\title{
Differential and integral invariants under Möbius transformation
}

\author{
He Zhang ${ }^{1,2, \star}$, Hanlin $\mathrm{Mo}^{1,2}$, You Hao ${ }^{1,2}$, Qi $\mathrm{Li}^{1,2}$, and Hua $\mathrm{Li}^{1,2}$ \\ 1 Key Laboratory of Intelligent Information Processing, Institute of Computing \\ Technology, Chinese Academy of Sciences, China \\ 2 University of Chinese Academy of Sciences, China \\ zhanghe@ict.ac.cn
}

\begin{abstract}
One of the most challenging problems in the domain of 2$\mathrm{D}$ image or 3-D shape is to handle the non-rigid deformation. From the perspective of transformation groups, the conformal transformation is a key part of the diffeomorphism. According to the Liouville Theorem, an important part of the conformal transformation is the Möbius transformation, so we focus on Möbius transformation and propose two differential expressions that are invariable under 2-D and 3-D Möbius transformation respectively. Next, we analyze the absoluteness and relativity of invariance on them and their components. After that, we propose integral invariants under Möbius transformation based on the two differential expressions. Finally, we propose a conjecture about the structure of differential invariants under conformal transformation according to our observation on the composition of above two differential invariants.
\end{abstract}

Keywords: Conformal transformation · Möbius transformation · Differential invariant · Integral invariant.

\section{Introduction}

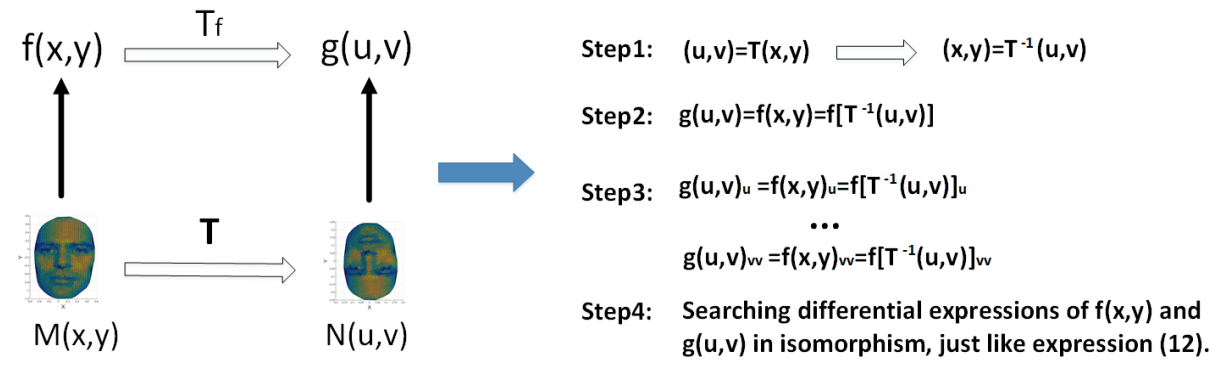

Fig. 1. A brief flowchart of the method.

One of the most challenging problems in the domain of 2-D image or 3$\mathrm{D}$ shape is to handle the non-rigid deformation, especially in the situation of

\footnotetext{
* Corresponding auther.
} 
anisotropy, which is universal in the real world. In the viewpoint of transformation groups, the isometric transformation is a prop subgroup of the conformal transformation, which is a prop subgroup of the diffeomorphism. Obviously, the anisotropic non-rigid transformation exceeds the boundary of isometric transformation and contains conformal transformation. Based on the Erlangen program of Klein, geometry is a discipline that studies the properties of space that remain unchanged under a particular group of transformation. In order to solve the anisotropic transformation problem, it is necessary to find the invariants under the conformal transformation.

The original motivation of conformal mapping is how to flatten the map of globe, and the Mercator projection produce an angle-preserving map that is very useful for navigation. More generally, the conformal geometry focuses on the shape in which the only measure is angle instead of usually length. The descriptions of conformal mapping contain angle preservation[12,26,5], metric rescaling[21,27], preservation of circles[14,28], etc. Some key ideas reside in the conformal surface geometry are Dirac equation[6], Cauchy-Riemann equation[22], Möbius transformations[27,28], Riemann mapping[10,9,35,33], Ricci flow[34], etc. The conformal geometry lies between the topology geometry and the Riemannian geometry, it studies the invariants of the conformal transformation group. The conformal structures [10,9] based on the theories of Riemann surfaces are invariants under conformal transformation. According to conformal geometry[7], the shape factor [10] and conformal module[35] are conformal invariants. Moreover, the conformal inner product[26] defined by an inner product of function is also changeless under conformal transformation. According to the Liouville Theorem[20,8], the Möbius transformation plays an important role in conformal mapping.

The definition of Möbius transformation[25] shows that it is compounded by a series of simple transformations: Translation, Stretching, Rotation, Reflection and Inversion. In the domain of invariants under translation, stretching and rotation transformations, the Geometric moment invariants(GMIs)[32] and the ShapeDNA[17] show a general method to generate the moment invariants; $\mathrm{Hu}$ et al[13] proposed a general construction method of surface isometric moment invariants based on the intrinsic metric. In the domain of invariants under reflection transformation, the chiral invariants[36] show the moment invariants based on the generating functions of ShapeDNA[17]. In the domain of invariants under conformal transformation, $\mathrm{Hu}[12]$ proposed limited conformal invariants based on geodesic tangent vectors. In the domain of invariants under Möbius transformation, the expression $\left(H^{2}-K\right) d A$ proposed by Blaschke[1] is proved to be a conformal invariant by Chen[4]; based on the Gauss-Bonnet Theorem, White[30] proposed that $\int_{M} H^{2} d A$ is a global conformal invariant if $M$ is an oriented and closed surface. The Gauss-Bonnet Theorem associates the differential expression(Gaussian curvature) of the surface $S$ with its topological invariant $\chi(S)$ (the Euler's characteristic). This great theorem motivates us to explore the differential invariants under the Möbius transformation since the differential expressions play essential roles in some procedures of physics, mathematics, computer sci- 
ence and other fields. In the domain of differential invariants, rotation and affine differential invariants were proposed by Olver[23] based on the moving frame method; a special type of affine differential invariants was presented by Wang et al[29]; Li et al[19] prove the existence of projective moment invariants of images with relative projective differential invariants; the research[18] on the relationship between differential invariants and moment invariants show that they are isomorphic under affine transformation.

In this article, we study invariants by combining functional map[24] and the derivatives of function(see Fig.1). In section 2, we show the background of this paper. In section 3, we propose the invariants under Möbius transformation. In section 4, we show another Möbius invariant from the functional view. Finally, we propose a conjecture about the structure of differential invariants under conformal transformation. The main contributions of this paper are as follows.

- We propose two differential expressions that are invariant under 2-D and 3D Möbius transformation respectively. According to the Liouville Theorem, the 3-D differential invariant is a conformal invariant.

- Based on the analysis on absoluteness and relativity of invariance about the two differential expressions and their components, we propose integral invariants under Möbius transformation.

- We propose a conjecture about the composition of differential invariants under conformal transformation.

\section{Notion and Background}

\section{$2.1 \quad$ Notion}

The formulation in this paper is same with the functional maps framwork[24]. Assuming $M$ and $N$ are two manifolds, a bijective mapping $T: M \rightarrow N$ induces the transformation $T_{F}: \mathcal{F}(M, \mathbb{R}) \rightarrow \mathcal{F}(N, \mathbb{R})$ of derived quantities, where $\mathcal{F}(\cdot, \mathbb{R})$ is scalar function defined on manifold. It means that any function $f: M \rightarrow \mathbb{R}$ have a counterpart function $g: N \rightarrow \mathbb{R}$ and $g=f \circ T^{-1}$.

To make the invariants under Möbius transformation clear, we partially modify original definition and theorem in this paper with this formulation.

\subsection{Theoretic Background}

According to the Liouville Theorem[20], the only conformal mapping in $R^{n}(n>$ 2) are Möbius transformation[11,25,15]. Furthermore, the Generalized Liouville Theorem shows that any conformal mapping defined on $D\left(D \in \overline{\mathbb{R}}^{n}, n>2\right)$ must be a restriction of Möbius transformation.

Theorem 1 (Generalized Liouville Theorem[8]). Suppose that $D, D^{\prime}$ are domains in $\overline{\mathbb{R}}^{n}$ and that $T: D \rightarrow D^{\prime}$ is a homeomorphism. If $n=2$, then $T$ is 1-quasiconformal if and only if $T$ or its complex conjucate is a meromorphic function of a complex variable in $D$. If $n \geq 3$, then $T$ is 1-quasiconformal if and only if $T$ is the restriction to $D$ of a Möbius transformation, i.e., the composition of a finite number of reflections in $(n-1)$-spheres and planes. 
Next, we will show the common expressions of Möbius transformation in different dimensions $(n \geq 2)$.

In the filed of complex analysis, a Möbius transformation could be expressed as

$$
T(z)=\frac{a z+b}{c z+d}
$$

where $a, b, c, d, z \in \mathbb{C}, a d-b c \neq 0$. Based on the Liouville Theorem[20], every Möbius transformation in higher dimensions could be given with the form

$$
T(x)=b+\frac{\gamma A(x-a)}{\|x-a\|_{2}^{\epsilon}},
$$

where $x, a, b \in \mathbb{R}^{n}, \epsilon$ is 0 or $2, \gamma \in \mathbb{R}$ and $A \in \mathbb{R}_{n \times n}$ is an orthogonal matrix. The choice of $\epsilon$ decides if $T(x)$ contains inversion transformation, and the sign of $\operatorname{det}(A)$ decides if $T(x)$ contains reflection transformation.

More generally, a Möbius transformation could be composed of a series of simple transformations, the definition of Möbius transformation is as below.

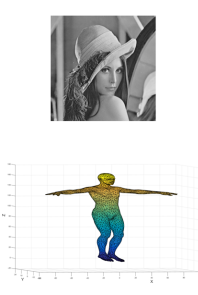

Original Data
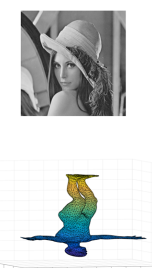

Reflection
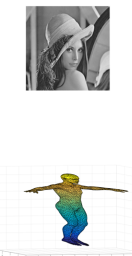

Stretching
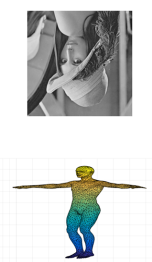

Rotation
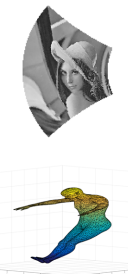

Inversion

Fig. 2. Some elementary transformations of Möbius transformation.

Definition 1 (Möbius transformation[25]). A n-dimension Möbius transformation is a homemorphism of $\overline{\mathbb{R}}^{n}$ (the one-point compactification of $\mathbb{R}^{n}$ ), it is a mapping $T: \overline{\mathbb{R}}^{n} \rightarrow \overline{\mathbb{R}}^{n}$ that is a finite composition of the following elementary transformations $\left(x \in \mathbb{R}^{n}\right)$ :

(1)Translation: $T_{a}(x)=x+a, a \in \mathbb{R}^{n}$.

(2) Stretching: $S_{s}(x)=s x, s \in \mathbb{R}$ and $s>0$.

(3)Rotation: $\operatorname{Rot}_{R}(x)=R x, R \in \mathbb{R}_{n \times n}$ and $R$ is an orthogonal matrix.

(4)Reflection about plane $P(a, t): \operatorname{Re} f_{a, t}(x)=x-2\left(a^{T} x-t\right) a, a \in \mathbb{R}^{n}$ is the normal vector of $P(a, t), t \in \mathbb{R}$ is the distance from the origin to $P(a, t)$.

(5)Inversion about sphere $S^{n-1}(a, r): I_{a, r}(x)=a+\frac{r^{2}(x-a)}{\|x-a\|_{2}^{2}}, a \in \mathbb{R}^{n}$ is the inversion center, $r$ is the inversion radius. 


\section{Möbius Invariants}

\subsection{Inversion Invariants}

In order to derive the differential invariant under inversion transformation $I_{a, r}$, in the 2-D situation we assume that the $T_{I_{a, r}}$ map the function $f(x, y)$ on domain $D \subset \overline{\mathbb{R}}^{n}$ to $g(u, v)$ on domain $D^{\prime} \subset \overline{\mathbb{R}}^{n}$, where $(u, v)=I_{a, r}(x, y)$ and $g(u, v)=$ $f(x, y)$, this means that the coordinates transformations under $I_{a, r}$ are as follows.

$$
\begin{aligned}
& u=a_{x}+\frac{r^{2}\left(x-a_{x}\right)}{\left(x-a_{x}\right)^{2}+\left(y-a_{y}\right)^{2}} \\
& v=a_{y}+\frac{r^{2}\left(y-a_{y}\right)}{\left(x-a_{x}\right)^{2}+\left(y-a_{y}\right)^{2}}
\end{aligned}
$$

At the same time, it means that the coordinates transformations under $I_{a, r}^{-1}$ are as follows.

$$
\begin{aligned}
& x=a_{x}+\frac{r^{2}\left(u-a_{x}\right)}{\left(u-a_{x}\right)^{2}+\left(v-a_{y}\right)^{2}} \\
& y=a_{y}+\frac{r^{2}\left(v-a_{y}\right)}{\left(u-a_{x}\right)^{2}+\left(v-a_{y}\right)^{2}}
\end{aligned}
$$

Based on $g(u, v)=f(x, y)$ and the equations (5)(6), we obtain the relationships between the partial derivatives of $g(u, v)$ and $f(x, y)$ as follows.

$$
\begin{aligned}
g_{u} & =f_{x} x_{u}+f_{y} y_{u} \\
g_{v} & =f_{x} x_{v}+f_{y} y_{v} \\
g_{u u} & =\left(f_{x x} x_{u}+f_{x y} y_{u}\right) x_{u}+f_{x} x_{u u}+\left(f_{y x} x_{u}+f_{y y} y_{u}\right) y_{u}+f_{y} y_{u u} \\
g_{u v} & =\left(f_{x x} x_{v}+f_{x y} y_{v}\right) x_{u}+f_{x} x_{u v}+\left(f_{y x} x_{v}+f_{y y} y_{v}\right) y_{u}+f_{y} y_{u v} \\
g_{v v} & =\left(f_{x x} x_{v}+f_{x y} y_{v}\right) x_{v}+f_{x} x_{v v}+\left(f_{y x} x_{v}+f_{y y} y_{v}\right) y_{v}+f_{y} y_{v v}
\end{aligned}
$$

Then we obtain a 2-D equation under the inversion transformation, it is

$$
\frac{g_{u u}+g_{v v}}{g_{u}^{2}+g_{v}^{2}}=\frac{f_{x x}+f_{y y}}{f_{x}^{2}+f_{y}^{2}}
$$

This means that

$$
\frac{f_{x x}+f_{y y}}{f_{x}^{2}+f_{y}^{2}}
$$

is a differential invariant under inversion transformation. We use the same method in 3-D situation and obtain a differential invariant under the inversion transformation, it is

$$
\frac{f_{A}+f_{B}}{\left(f_{x}^{2}+f_{y}^{2}+f_{z}^{2}\right)^{2}}
$$

where

$$
\begin{aligned}
& f_{A}=\left(f_{x x}+f y y+f z z\right)\left(f_{x}^{2}+f_{y}^{2}+f_{z}^{2}\right) \\
& f_{B}=f_{x}^{2} f_{x x}+f_{y}^{2} f_{y y}+f_{z}^{2} f_{z z}+2 f_{x} f_{x y} f_{y}+2 f_{x} f_{x z} f_{z}+2 f_{y} f_{y z} f_{z}
\end{aligned}
$$




\subsection{The Boundary of Invariance}

We have shown that (13) and (14) are differential invariants under inversion transformation. It is obvious that they are invariants under translation transformation. We prove that (13) and (14) are also differential invariants under rotation, stretching and reflection transformations(see Appendix A for a proof). According to the definition of Möbius transformation, we conclude that the differential expression (13) is a differential invariant under 2-D Möbius transformation. Furthermore, with the Generalized Liouville Theorem we obtain that (14) is a conformal invariant.

\subsection{Absoluteness and Relativity of Invariance}

If expression $\operatorname{Inv}_{T}$ is an invariant under transformation $T$, the transformed expression $\operatorname{Inv}_{T}^{\prime}$ satisfies

$$
\operatorname{Inv}_{T}^{\prime}=W_{T} \cdot \operatorname{Inv}_{T}
$$

where $W_{T}$ is an expression related to $T$. In this context, $\operatorname{Inv}_{T}$ is an absolute invariant if $W_{T} \equiv 1$, otherwise, $I n v_{T}$ is a relative invariant. Base on the analysis in $3.2,(13)$ is an absolute invariant under Möbius transformation and (14) is an absolute invariant under conformal transformation. Next, we will show the numerator and denominator of (13) or (14) are relative invariants.

In the derivation of 2-D inversion invariants, we obtain that $W_{I_{a, r}}=\|J\|^{-1}$ for the numerator and denominator of (13), this means

$$
\begin{gathered}
g_{u u}+g_{v v}=\|J\|^{-1}\left(f_{x x}+f_{y y}\right) \\
g_{u}^{2}+g_{v}^{2}=\|J\|^{-1}\left(f_{x}^{2}+f_{y}^{2}\right)
\end{gathered}
$$

where $|J|$ is the determinant of Jacobian matrix of transformation $I_{a, r},\|J\|$ is the absolute valve of $|J|$. In 3 -D situation, we obtain $W_{I_{a, r}}=\|J\|^{-\frac{4}{3}}$ for the numerator and denominator of (14). In the stretching transformation, we obtain $W_{S}=\|J\|^{-1}$ in 2-D situation, and $W_{S}=\|J\|^{-\frac{4}{3}}$ in 3-D situation. We also obtain that $W_{T}=1$ for the numerator and denominator of (13) or (14) under translation, rotation and reflection transformations.

The result of absoluteness and relativity of invariance on (13) and (14) is shown in Table 1.

\subsection{Multiscale and Quantity}

Assuming $f(x, y)$ is a regular parameter surface $S$ defined on $D$, if $T_{F}$ transform $f(x, y)$ defined on $D$ to $g(u, v)$ defined on $D^{\prime}$ and $g(u, v)=f(x, y)$, based on the change of variable theorem[16] for multiple integrals and Table 1 we obtain that

$$
\iint_{D^{\prime}}\left(g_{u u}+g_{v v}\right) d u d v=\iint_{D} W_{T}\left(f_{x x}+f_{y y}\right)\left\|J_{T}\right\| d x d y=\iint_{D}\left(f_{x x}+f_{y y}\right) d x d y
$$


Table 1. The form of $W_{T}$ under Transformations

\begin{tabular}{|c|c|c|c|c|c|}
\hline Expression & Translation & Stretching & Rotation & Reflection & Inversion \\
\hline (13) and (14) & 1 & 1 & 1 & 1 & 1 \\
Num $^{a} /$ Den of (13) & 1 & $\|J\|^{-1}$ & 1 & 1 & $\|J\|^{-1}$ \\
Num $/$ Den $^{b}$ of (14) & 1 & $\|J\|^{-\frac{4}{3}}$ & 1 & 1 & $\|J\|^{-\frac{4}{3}}$ \\
\hline
\end{tabular}

${ }^{a}$ Num means the numerator of fraction .

${ }^{b}$ Den means the denominator of fraction.

$$
\iint_{D^{\prime}}\left(g_{u}^{2}+g_{v}^{2}\right) d u d v=\iint_{D} W_{T}\left(f_{x}^{2}+f_{y}^{2}\right)\left\|J_{T}\right\| d x d y=\iint_{D}\left(f_{x}^{2}+f_{y}^{2}\right) d x d y
$$

where $\left\|J_{T}\right\|$ is the area extension factor, so we obtain that

$$
\begin{gathered}
\iint_{D}\left(f_{x x}+f_{y y}\right) d x d y \\
\iint_{D}\left(f_{x}^{2}+f_{y}^{2}\right) d x d y
\end{gathered}
$$

are integral invariants under 2-D Möbius transformation. In the same way, we obtain that

$$
\begin{gathered}
\iiint_{D}\left(f_{x}^{2}+f_{y}^{2}+f_{z}^{2}\right)^{\frac{3}{2}} d x d y d z \\
\iiint_{D}\left(f_{A}+f_{B}\right)^{\frac{3}{4}} d x d y d z
\end{gathered}
$$

are integral invariants under 3-D conformal transformation.

Actually a differential expression $I n v_{T}$ of function $f$ defined on domain $D_{f}$ accurately characterize $f$ at point of $D_{f}$, it provides extremely wide space to describe the function $f$.

Multiscale of Invariants Assuming $F_{i}\left(\operatorname{Inv}_{T}\right)$ is a function of $\operatorname{Inv}_{T}$, a general method to construct descriptors in different scale is the integral of $\int_{D_{j}} F_{i}\left(\operatorname{Inv}_{f}\right) d A$ on region $D_{j}\left(D_{j} \subset D_{f}\right)$ with different size, and when $D_{j}=D_{f}$ the result is a global invariant, for example, the Willmore energy $\int\left(H^{2}-K\right) d A[1]$ applied in the theory of surfaces[31], digital geometry processing[2] and other fields.

In this view, the only difference between invariant with specify-scale and global invariant is the definition domain, the construction method of specifyscale invariant is same with global invariant. The former could be elaborately modified by selecting domain of integration in different applications.

Quantity of Invariants A general method to construct a large number of invariants is using various functions $F_{i}\left(I n v_{T}\right)$ with these functions are independent of each other[3]. We just show a simple method to construct integral invariants based on differential invariants and integral, in addition, more invariant forms 
can be constructed with differential invariants. Next, we give a possible form of invariants under Möbius transformation:

$$
\begin{gathered}
\iint_{D} \frac{\left(f_{x x}+f_{y y}\right)^{n+1}}{\left(f_{x}^{2}+f_{y}^{2}\right)^{n}} d x d y \\
\iint_{D} \frac{\left(f_{x}^{2}+f_{y}^{2}\right)^{n+1}}{\left(f_{x x}+f_{y y}\right)^{n}} d x d y \\
\iiint_{D} \frac{\left(f_{A}+f_{B}\right)^{\frac{3}{4}(n+1)}}{\left(f_{x}^{2}+f_{y}^{2}+f_{z}^{2}\right)^{\frac{3}{2} n}} d x d y d z \\
\iiint_{D} \frac{\left(f_{x}^{2}+f_{y}^{2}+f_{z}^{2}\right)^{\frac{3}{2}(n+1)}}{\left(f_{A}+f_{B}\right)^{\frac{3}{4} n}} d x d y d z
\end{gathered}
$$

if the denominators of (25), (26), (27), (28) are not zero.

\subsection{Another Conformal Invariant}

The expression $\left(H^{2}-K\right) d A$ proposed by Biacchke[1] has been proved to be an invariant under Möbius transformation[4,30]. It differs from our method in two important respects: the domain of transformation and the number of functions participated in invariants(see detailed expression at Appendix B).

\section{Conjecture of Conformal Invariants}

We have shown that (13) is a Möbius invariant and (14) is a conformal invariant. However, the fascinating part of (13) or (14) is that the differential expressions

$$
\begin{gathered}
f_{x}^{2}+f_{y}^{2} \quad \text { or } f_{x}^{2}+f_{y}^{2}+f_{z}^{2} \\
f_{x x}+f_{y y} \quad \text { or } f_{x x}+f_{y y}+f_{z z} \\
f_{x}^{2} f_{x x}+f_{y}^{2} f_{y y}+f_{z}^{2} f_{z z}+2 f_{x} f_{x y} f_{y}+2 f_{x} f_{x z} f_{z}+2 f_{y} f_{y z} f_{z}
\end{gathered}
$$

are differential invariants under rigid transformation. Based on this observation and the fact that the differential expressions play important roles in transformation, we have a bold conjecture about the structure of differential invariants under conformal transformation.

Conjecture: The differential invariants under conformal transformation are composed of differential invariants under rigid transformation in a self-consistent manner.

One of the possible self-consistent forms in n-dimensional Euclidean space may be

$$
\sum_{i=1}^{n-1} \frac{\prod_{j=1}^{a_{i}} D R I_{j}}{\left(f_{x_{1}}^{2}+f_{x_{2}}^{2}+\cdots+f_{x_{n}}^{2}\right)^{n-1}}
$$

where $D R I$ is differential invariant under rigid transformation. 


\section{Experimental Results}

We choose a human face model from TOSCA database and treat the $\mathrm{z}$-coordinate value of vertexes of the triangle mesh as a function $f$ defined on $\mathrm{x}$-coordinate and y-coordinate, i.e. $z=f(x, y)$. With least square method, the coordinates of a vertice and its 1-ring neighbors were used to estimate parameters in Taylor expansion of $f$ at the vertice; in order to guarantee the accuracy of descriptor calculation, we only consider vertexes that are located inside the mesh and have enough 1-ring neighbors. After that, we calculate a descriptor at the vertice and the descriptor is composed by (13), (25) and (26) with different $n(\geq 0)$. Moreover, in integral invariants, the area $A_{\text {vert }}$ around a vertice is determined by Mixed Voronoi cell.

We deform the definition domain of $f$ with reflection, stretching, rotation and inversion transformation(Fig.3). In reflection transformation, $a=(1,0)$ and $t=0$; the $s$ in stretching transformation is 2 ; in rotation transformation the original data is rotated 90 degrees counterclockwise; in inversion transformation the inversion center is $(0,1000)$ and inversion radius is 500 (see more explanation about experiments at Appendix C).

\subsection{Stability of Invariants}

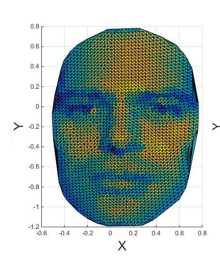

Original Data

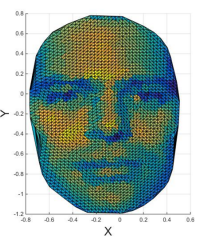

Reflection

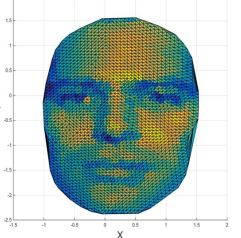

Stretching

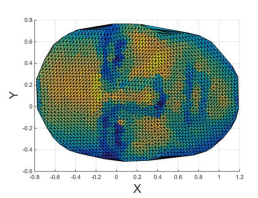

Rotation

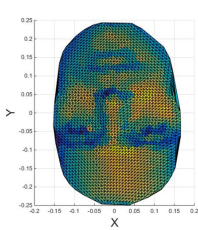

Inversion

Fig. 3. Elementary transformations of Möbius transformation on human face model.

In this experiment we choose $n=0,1$ and the integral invariants is calculated at the local area of each vertex. After we obtain a 5-dimension descriptor at vertexes of the five mesh in Fig.3, we calculate the average error of each dimension of the descriptor. In addition, we choose an isometric invariant at the vertex, the Laplacian operator, to compare with above invariants. The average error of each dimension is calculated by the following formula

$$
E r r=\frac{1}{N} \sum_{i} \frac{\left|\operatorname{Inv}_{T ; i}-\operatorname{Inv}_{O ; i}\right|}{\left|\operatorname{Inv_{T;i}|}\right|+\left|\operatorname{Inv}_{O ; i}\right|} \times 100 \%
$$

where $\operatorname{In} v_{O ; i}$ is the value of invariant at vertex $i$ on original data, $I n v_{T ; i}$ is the value of invariant at vertex $i$ on deformed data, and $N$ is the total number of vertexes participated in the calculation. The result of this experiment is in Table 2, it shows that (13), (25) and (26) are invariants under Möbius transformations. 
Table 2. The average error of Laplacian operator and Möbius invariants.

\begin{tabular}{|c|c|c|c|c|}
\hline Expression & Reflection & Stretching & Rotation & Inversion \\
\hline$f_{x x}+f_{y y}$ & 0 & $6.00 \times 10^{1}$ & $4.82 \times 10^{-13}$ & $8.82 \times 10^{1}$ \\
\hline$\frac{f_{x x}+f_{y y}}{f_{x}^{2}+f_{y}^{2}}$ & 0 & $1.20 \times 10^{-12}$ & $1.33 \times 10^{-12}$ & $1.98 \times 10^{-3}$ \\
\hline $\iint_{D}\left(f_{x x}+f_{y y}\right) d x d y$ & 0 & $4.38 \times 10^{-13}$ & $4.82 \times 10^{-13}$ & $1.69 \times 10^{-1}$ \\
\hline $\iint_{D}\left(f_{x}^{2}+f_{y}^{2}\right) d x d y$ & 0 & $1.21 \times 10^{-12}$ & $1.27 \times 10^{-12}$ & $1.69 \times 10^{-1}$ \\
\hline $\iint_{D} \frac{\left(f_{x x}+f_{y y}\right)^{2}}{f_{x}^{2}+f_{y}^{2}} d x d y$ & 0 & $1.24 \times 10^{-12}$ & $1.47 \times 10^{-12}$ & $1.70 \times 10^{-1}$ \\
\hline $\iint_{D} \frac{\left(f_{x}^{2}+f_{y}^{2}\right)^{2}}{f_{x x}+f_{y y}} d x d y$ & 0 & $2.39 \times 10^{-12}$ & $2.58 \times 10^{-12}$ & $1.70 \times 10^{-1}$ \\
\hline
\end{tabular}

\subsection{Discrimination of Invariants}

In this experiment we use the 5-dimension descriptor of vertex at original to match its corresponding vertex in the deformed mesh with nearest neighbor rule, the metric between vertexes is standardized Euclidean distance. The error rate (percentage) of this experiment is in Table 3.

Table 3. The error rate (percentage) of Möbius invariants in vertex matching.

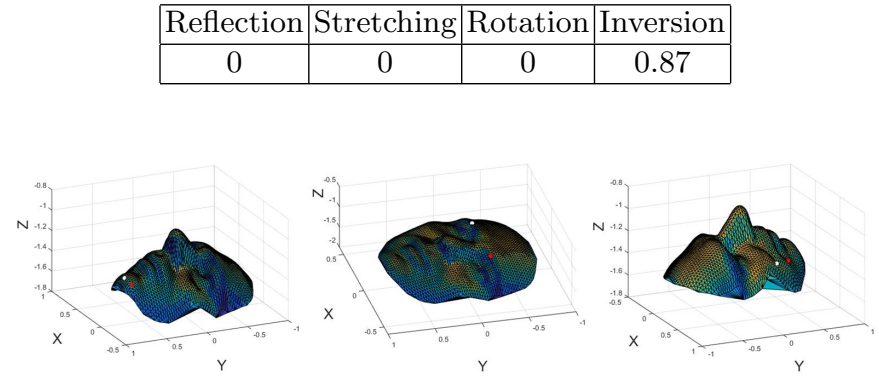

Fig. 4. Some situations where vertex matching fails.

In conformal deformation scenario, this experiment shows the potential of Möbius invariants in matching task. Fig.4 shows some matching-fail situations, where the white point is the real position and the red point is the matching vertex. The reason for most matching failures is that the original white vertex and deformed red vertex have similar functional distribution environments.

\section{Conclusions}

In this article, we propose two differential invariants under 2-D and 3-D Möbius transformation respectively, in particular, the 3-D expression is a conformal invariant according to the Liouville Theorem. After that, we analyze the absoluteness and relativity of invariance on the two expressions and their components, 
and we show an integral construction method that targets to the multiscale and quantity of invariant, the experimental results show that the invariants proposed in this paper perform well. Furthermore, we show another Möbius invariant from the functional view. Finally, we propose a conjecture about the structure of differential invariants under conformal transformation.

This article shows a method of combining functional map and derivatives of function to study conformal invariant, more research about the differential invariants under conformal transformation is necessary in the future. In addition to practical application solutions based on Möbius invariants, questing the generative structure of conformal differential invariant is also an interesting topic.

\section{Acknowledgment}

The authors would like to thank Dr. Antti Rrasila of Aalto University for providing help on how to distinguish Möbius invariants and conformal invariants.

This work was partly funded by National Key R\&D Program of China (No. 2017YFB1002703) and National Natural Science Foundation of China (Grant No.60873164, 61227802 and 61379082).

\section{References}

1. Biaschke, W.: Vorlesungen über differentialgeometrie iii (1929)

2. Bobenko, A.I., Schröder, P.: Discrete willmore flow (2005)

3. Brown, A.B.: Functional dependence. Transactions of the American Mathematical Society 38(2), 379-394 (1935)

4. Chen, B.Y.: An invariant of conformal mappings. Proceedings of the American Mathematical Society 40(2), 563-564 (1973)

5. Corman, E., Solomon, J., Ben-Chen, M., Guibas, L., Ovsjanikov, M.: Functional characterization of intrinsic and extrinsic geometry. ACM Transactions on Graphics (TOG) 36(2), 14 (2017)

6. Crane, K., Pinkall, U., Schröder, P.: Spin transformations of discrete surfaces. ACM Transactions on Graphics (TOG) 30(4), 104 (2011)

7. Farkas, H.M., Kra, I.: Riemann surfaces. In: Riemann surfaces, pp. 9-31. Springer (1992)

8. Gehring, F.: Topics in quasiconformal mappings. In: Quasiconformal Space Mappings, pp. 20-38. Springer (1992)

9. Gu, X., Wang, Y., Yau, S.T., et al.: Computing conformal invariants: Period matrices. Communications in Information \& Systems 3(3), 153-170 (2003)

10. Gu, X., Yau, S.T.: Surface classification using conformal structures. In: null. p. 701. IEEE (2003)

11. Haantjes, J.: Conformal Representation of an N-dimensional Euclidean Space with a Non-definite Fundamental Form on Itself (1937)

12. Hu, P.: A Class of Isometric Invariants and Their Applications(in Chinese). Ph.D. thesis, Institute of Computing Technology, Chinese Academy of Sciences (May 2011)

13. Hu, P., Li, H., Lin, Z.: A construction method for surface isometric invariants. Journal of Systems Science and Mathematical Sciences 9, 006 (2009) 
14. Kharevych, L., Springborn, B., Schröder, P.: Discrete conformal mappings via circle patterns. ACM Transactions on Graphics (TOG) 25(2), 412-438 (2006)

15. Kühnel, W., Rademacher, H.B.: Liouville's theorem in conformal geometry. Journal de mathématiques pures et appliquées 88(3), 251-260 (2007)

16. Lax, P.D.: Change of variables in multiple integrals. The American mathematical monthly 106(6), 497-501 (1999)

17. Li, E., Huang, Y., Xu, D., Li, H.: Shape dna: Basic generating functions for geometric moment invariants. arXiv preprint arXiv:1703.02242 (2017)

18. Li, E., Li, H.: Isomorphism between differential and moment invariants under affine transform. arXiv preprint arXiv:1705.08264 (2017)

19. Li, E., Mo, H., Xu, D., Li, H.: Image projective invariants. IEEE Transactions on Pattern Analysis and Machine Intelligence (2018)

20. Liouville, J.: Extension au cas des trois dimensions de la question du tracé géographique. Applications de lanalyse à la géométrie pp. 609-617 (1850)

21. Luo, F.: Combinatorial yamabe flow on surfaces. Communications in Contemporary Mathematics 6(05), 765-780 (2004)

22. Mullen, P., Tong, Y., Alliez, P., Desbrun, M.: Spectral conformal parameterization. Computer Graphics Forum 27(5), 1487-1494 (2008)

23. Olver, P.J.: Equivalence, invariants and symmetry. Cambridge University Press (1995)

24. Ovsjanikov, M., Ben-Chen, M., Solomon, J., Butscher, A., Guibas, L.: Functional maps: a flexible representation of maps between shapes. ACM Transactions on Graphics (TOG) 31(4), 30 (2012)

25. Rasila, A.: Introduction to quasiconformal mappings in n-space. Proceedings of the International Workshop on Quasiconformal (2006)

26. Rustamov, R.M., Ovsjanikov, M., Azencot, O., Ben-Chen, M., Chazal, F., Guibas, L.: Map-based exploration of intrinsic shape differences and variability. ACM Transactions on Graphics (TOG) 32(4), 72 (2013)

27. Springborn, B., Schröder, P., Pinkall, U.: Conformal equivalence of triangle meshes. ACM Transactions on Graphics (TOG) 27(3), 77 (2008)

28. Vaxman, A., Müller, C., Weber, O.: Conformal mesh deformations with möbius transformations. ACM Transactions on Graphics (TOG) 34(4), 55 (2015)

29. Wang, Y., Wang, X., Zhang, B.: Affine differential invariants of functions on the plane. Journal of Applied Mathematics 2013 (2013)

30. White, J.H.: A global invariant of conformal mappings in space. Proceedings of the American Mathematical Society 38(1), 162-164 (1973)

31. Willmore, T.J.: Surfaces in conformal geometry. Annals of Global Analysis and Geometry 18(3-4), 255-264 (2000)

32. Xu, D., Li, H.: Geometric moment invariants. Pattern recognition 41(1), 240-249 (2008)

33. Xu, J., Kang, H., Chen, F.: Content-aware image resizing using quasi-conformal mapping. The Visual Computer 34(3), 431-442 (2018)

34. Yu, X., Lei, N., Wang, Y., Gu, X.: Intrinsic 3d dynamic surface tracking based on dynamic ricci flow and teichmüller map. Proceedings. IEEE International Conference on Computer Vision 2017, 5400-5408 (2017)

35. Zeng, W., Gu, X.D.: Registration for $3 \mathrm{~d}$ surfaces with large deformations using quasi-conformal curvature flow. Computer Vision and Pattern Recognition (CVPR) (2011)

36. Zhang, H., Mo, H., Hao, Y., Li, S., Li, H.: Fast and efficient calculations of structural invariants of chirality. arXiv preprint arXiv:1711.05866 (2017) 